Теорія Ймовір. та Матем. Статист. Вип. 79, 2008
Theor. Probability and Math. Statist.

No. 79, 2009, Pages 171-178

S 0094-9000(09)00789-3

Article electronically published on December 30, 2009

\title{
ON THE RATE OF CONVERGENCE OF PRICES OF BARRIER OPTIONS WITH DISCRETE AND CONTINUOUS TIME
}

UDC 519.21

\author{
O. M. SOLOVEYKO AND G. M. SHEVCHENKO
}

\begin{abstract}
A barrier option is a derivative realized or cancelled if the price of the underlying asset crosses a certain barrier. Most of the models in financial mathematics are considered for markets with continuous time. However the trading days for a particular stock take place at separate moments, i.e. discretely. The Black-Scholes model is extended in the paper in the sense that we consider barrier options with varying drifts. We find the rate of convergence of prices of such options with discrete time to the prices of options with continuous time.
\end{abstract}

\section{INTRODUCTION}

A barrier option is a derivative security, whose payoff depends on whether or not the trajectory of the underlying asset price crosses a predetermined barrier level over a predetermined time.

Therefore the payoff depends on the complete history of the option price during a certain time period and a barrier option is thus a particular case of exotic options.

There are two types of barrier call and put options, namely knock-out and knock-in options. The two main types of knock-out options are 1) down-and-out options and 2) up-and-out options. A down-and-out option gives the holder the right but not the obligation to buy (in the case of a call) or sell (in the case of a put) shares of an underlying asset at a predetermined strike price so long as the price of that asset did not go below a predetermined barrier during the option lifetime. That is, once the price of the underlying asset falls below the barrier, the option is knocked out and no longer carries any value.

An up-and-out option is similar to a down-and-out option, the only difference being the placement of the barrier. Rather than being knocked-out by falling below the barrier price, up-and-out options are knocked-out if the price of the underlying asset rises above the predetermined barrier.

Similarly there are two types of knock-in options, namely up-and-in and down-and-in options. A down-and-in option is the opposite of a down-and-out barrier option. Downand-in options only carry a value if the price of the underlying asset falls below the barrier price during the option lifetime.

In total, there are eight types of barrier options.

For example, the payoff function of an up-and-in option is given by

$$
C= \begin{cases}\left(S_{T}-K\right)^{+} & \text {if } \max _{0 \leq t \leq T} S_{t} \geq H \\ 0 & \text { otherwise }\end{cases}
$$

2000 Mathematics Subject Classification. Primary 91B28; Secondary 60G50, 60F05. 
where $H$ is the barrier level $\left(H>S_{0}\right.$ and $\left.H>K\right)$ and $K$ is the strike price. The payoff functions for other types of barrier options are defined similarly.

The problem of estimating and hedging the barrier options in models with continuous time is very complicated and the prices for such options can be expressed analytically only in the simplest cases. Thus one requires an approximated estimation of such options. The simplest of numerical methods is the time discretization method that can be described as follows. The time interval is split into $m$ parts of equal length and a price model is studied for the discrete time. For this setting, the price of an option can be evaluated approximately with the help of the Monte Carlo method by simulating the trajectory of the price of the underlying asset. On the other hand, one may be interested in solving an opposite problem: if one knows an analytical formula for the price of an option in a continuous time model, then one may need to estimate an option activated in the event when the price of the underlying asset crosses a predetermined barrier level if the price is observed at discrete time instances (for example, at the end of every trading day).

From the point of view of a practitioner, it is important to know the quality of the approximated estimation of an option, that is, an order of magnitude of the error.

The approximated option prices with an error $o\left(m^{-1 / 2}\right)$ are obtained in [1] for barrier options of four types for a model with constant parameters by using a compensating factor.

Theorem $1.1([1)$. Let $V(H)$ be the price of a barrier option in the continuous time model and let $V_{m}(H)$ be the price of the same barrier option in the discrete time model $0, T / m, 2 T / m, \ldots, T$. Then

$$
V_{m}(H)=V(H \exp \{ \pm \beta \sigma \sqrt{T / m}\})+o\left(m^{-1 / 2}\right)
$$

for every discrete time barrier option, where the symbol "+" is applied to the "up" option and the symbol "-" is applied for the "down" option. Here $\beta=-(\zeta(1 / 2) / \sqrt{2 \pi}) \approx 0.5826$ and $\zeta$ denotes the Riemann zeta function.

A short proof of this result is given in [7. Note that this result is proved in [7] as well as in [5] for all eight types of barrier options. The rate of convergence for lookback options and other exotic options is obtained in 2 .

In this paper, we study the question on how close each to other are the prices of barrier options with discrete and continuous time in the case where the price of the asset is modelled by the geometric Brownian motion with a varying drift coefficient. We prove that the rate of convergence of prices for such options is of order $O\left(m^{-1 / 2}\right)$.

\section{MAin Results}

Let $(\Omega, \mathcal{F}, \mathrm{P})$ be a complete probability space with a filtration $\left\{\mathcal{F}_{t}, t \geq 0\right\}$ and let the stochastic process $\left\{W_{t}, t \geq 0\right\}$ be a standard $\mathcal{F}_{t}$-Brownian motion defined on this probability space. Consider the Black-Scholes model of the financial market involving two assets. One of these assets is riskless whose price at the moment $t$ is given by

$$
B_{t}=B_{0} \exp \left\{\int_{0}^{t} r_{s} d s\right\} .
$$

The other asset is risky and its price is described by

$$
S_{t}=S_{0} \exp \left\{\int_{0}^{t} \mu_{s} d s+\sigma W_{t}\right\} .
$$

For simplicity, we assume that $\mathrm{P}$ is a martingale measure for the discounted price process of the risky asset, namely $\mu_{t}=r_{t}-\sigma^{2} / 2$. 
Moreover, we assume the Lipschitz condition for the interest rate $r_{t}$, that is,

$$
\left|r_{t}-r_{s}\right| \leq C|t-s|
$$

for all $t, s \in[0, T]$, where $C$ is a certain constant.

In the financial market with continuous time, the fair price of an option is defined as the mathematical expectation of the discounted payoff for this option with respect to the martingale measure. Let $I_{A}$ denote the indicator of an event $A$ and let $x^{+}=\max \{x, 0\}$. For an arbitrary process $Y(t)$, we put $\tau(x, Y):=\inf \{t \geq 0: Y(t) \geq x\}$. In other words, $\tau(H, S)=\tau_{H}$ is the first time $t$ when $S_{t}$ reaches the level $H$. Then, for example, the price of a European call "up-and-out" option is given by

$$
V(H)=\mathrm{E}\left(\exp \left\{-\int_{0}^{T} r_{t} d t\right\}\left(S_{T}-K\right)^{+} I_{\{\tau(H, S)>T\}}\right),
$$

where $K>0$ is the strike price and $H>S_{0}$ is a barrier level. Similarly, the price of a European put "down-and-in" option is

$$
V(H)=\mathrm{E}\left(\exp \left\{-\int_{0}^{T} r_{t} d t\right\}\left(K-S_{T}\right)^{+} I_{\left\{\tau^{*}(H, S) \leq T\right\}}\right),
$$

where $H<S_{0}$ is the barrier level and $\tau^{*}(x, Y):=\inf \{t \geq 0: Y(t) \leq x\}$. The prices of other barrier options are determined in the same way. Merton [8] found explicitly the prices of all eight types of barrier options for the case where the riskless interest rate $r$ is constant.

Now we consider a discrete time model. We split the time interval $[0, T]$ into $m$ parts by the points $t_{i}=i \Delta t, i=0, \ldots, m$. At the point $t_{n}$, we define the price of a share by

$$
S_{n}=S_{n-1} \exp \left\{\mu_{t_{n}} \Delta t+\sigma \sqrt{\Delta t} Z_{n}\right\}, \quad n \geq 1 .
$$

Here the role of the "random force" of a financial market, which is played by Brownian motion in the continuous time model, is played by the random walk $W_{n}$ defined by

$$
W_{n}:=\sum_{i=1}^{n} Z_{i}
$$

where the $Z_{i}$ are independent standard normal random variables. Now the price of a European call "up-and-out" is defined by

$$
V_{m}(H)=\mathrm{E}\left(\exp \left\{-\sum_{i=0}^{m-1} r_{t_{i}} \Delta t\right\}\left(S_{m}-K\right)^{+} I_{\left\{\tau^{\prime}(H, S)>m\right\}}\right),
$$

where $\tau^{\prime}(H, S):=\inf \left\{n \geq 1: S_{n} \geq H\right\}$; that is, $\tau^{\prime}(H, S)=\tau_{H}^{m}$ is the first discrete moment $k$ when $S_{t_{k}}$ crosses the level $H$.

In what follows the symbol $C$ denotes a generic constant whose value may change from one line to another.

Theorem 2.1. Let the continuous time model for the price of a risky asset be as described above. Let condition (2.1) hold. The difference between the fair prices of a European call "up-and-out" option for the discrete and continuous time is such that

$$
V_{m}(H)-V(H)=O\left(\frac{1}{\sqrt{m}}\right), \quad m \rightarrow \infty .
$$

Proof. Since the joint distribution of random variables $\Delta t W_{1}, \ldots, \Delta t W_{m}$ coincides with that of $W_{t_{1}}, \ldots, W_{t_{m}}$, one can assume that $W_{n}=W_{t_{n}}$. This assumption simplifies any further reasoning. 
Consider the difference between the fair prices of a European call "up-and-out" option for the discrete and continuous models:

$$
\begin{aligned}
V_{m}(H)-V(H)= & \exp \left\{-\sum_{i=0}^{m-1} r_{t_{i}} \Delta t\right\} \mathrm{E}\left(\left(\left(S_{m}-K\right)^{+}-\left(S_{T}-K\right)^{+}\right) I_{\left\{\tau_{H}^{m}>T\right\}}\right) \\
& +\exp \left\{-\sum_{i=0}^{m-1} r_{t_{i}} \Delta t\right\} \mathrm{E}\left(\left(S_{T}-K\right)^{+}\left(I_{\left\{\tau_{H}^{m}>T\right\}}-I_{\left\{\tau_{H}>T\right\}}\right)\right) \\
& +\mathrm{E}\left(\left(S_{T}-K\right)^{+} I_{\left\{\tau_{H}^{m}>T\right\}}\right)\left(\exp \left\{-\sum_{i=0}^{m-1} r_{t_{i}} \Delta t\right\}-\exp \left\{-\int_{0}^{T} r_{t} d t\right\}\right) \\
= & \delta_{1}^{m}+\exp \left\{-\sum_{i=0}^{m-1} r_{t_{i}} \Delta t\right\} \mathrm{E}\left(\left(S_{T}-K\right)^{+}\left(I_{\left\{\tau_{H}^{m}>T\right\}}-I_{\left\{\tau_{H}>T\right\}}\right)\right)+\delta_{2}^{m},
\end{aligned}
$$

where

$$
\begin{aligned}
\left|\delta_{2}^{m}\right| & \leq(H-K)\left|\left(\exp \left\{-\int_{0}^{T} r_{t} d t\right\}-\exp \left\{-\sum_{i=0}^{m-1} r_{t_{i}} \Delta t\right\}\right) \mathrm{P}\left(\tau_{H}^{m}>T\right)\right| \\
& \leq C\left|\exp \left\{-\int_{0}^{T} r_{t} d t+\sum_{i=0}^{m-1} r_{t_{i}} \Delta t\right\}-1\right|
\end{aligned}
$$

by the assumptions imposed on the function $r$.

Since $|\exp \{x\}-1| \leq|x| \exp \{|x|\}$ for arbitrary $x$,

$$
\begin{aligned}
\left|\delta_{2}^{m}\right| & \leq C\left|-\int_{0}^{T} r_{t} d t+\sum_{i=0}^{m-1} r_{t_{i}} \Delta t\right|=C\left|\sum_{i=0}^{m-1} \int_{t_{i}}^{t_{i+1}}\left(r_{t}-r_{t_{i}}\right) d t\right| \\
& \leq C \sum_{i=0}^{m-1} \int_{t_{i}}^{t_{i+1}}\left|r_{t}-r_{t_{i}}\right| d t \leq C \Delta t \leq C \sqrt{\Delta t}
\end{aligned}
$$

To estimate $\delta_{1}^{m}$ note that $S$ is a solution of the stochastic differential equation

$$
d S_{t}=r_{t} d t+\sigma d W_{t}
$$

and $S_{m}$ is the Euler approximation for the value $S_{T}$ of the latter solution. Then, due to a well-known estimate of their difference (see e.g. [6, Section 9.6]) and the boundedness of $r$,

$$
\left|\delta_{1}^{m}\right| \leq C \mathrm{E}\left|S_{T}-S_{m}\right| \leq C \sqrt{\Delta t}
$$

Thus

$$
\begin{aligned}
\left|V_{m}(H)-V(H)\right| & \leq C\left|\mathrm{E}\left((H-K)\left(I_{\left\{\tau_{H}^{m}>T\right\}}-I_{\left\{\tau_{H}>T\right\}}\right)\right)\right|+C \sqrt{\Delta t} \\
& =C\left|\mathrm{E}\left(I_{\left\{\tau_{H}^{m}>T\right\}}-I_{\left\{\tau_{H}>T\right\}}\right)\right|+C \sqrt{\Delta t} .
\end{aligned}
$$

To estimate the first term we apply the Girsanov formula. First we introduce the following notation. For a bounded measurable function $g:[0, T] \rightarrow \mathbf{R}$, let

$$
W_{t}(g)=W_{t}+\int_{0}^{t} \frac{g(s)}{\sigma} d s
$$

be a Brownian motion with drift $g / \sigma$,

$$
\mathrm{E}(g)=\exp \left\{-\int_{0}^{T} \frac{g(s)}{\sigma} d W_{s}-\frac{1}{2} \int_{0}^{T} \frac{g^{2}(s)}{\sigma^{2}} d s\right\}
$$


be the density of the martingale measure $P^{g}$ for $W(g)$, and $\mathrm{E}^{g}(\cdot)=\mathrm{E}(\mathrm{E}(g) \cdot)$ be the mathematical expectation with respect to this measure. For a process $X$, let

$$
X^{*}=\sup _{[0, T]} X_{t}, \quad X_{m}^{*}=\max _{1 \leq i \leq m} X_{t_{i}} .
$$

We also put

$$
\mu_{t}^{m}=\sum_{i=0}^{m-1} \mu_{t_{i}} I_{t \in\left[t_{i}, t_{i+1}\right)}, \quad L=\frac{1}{\sigma} \ln \left(H / S_{0}\right), \quad S_{m}^{\prime}=\max _{1 \leq i \leq m} S_{i} .
$$

According to Girsanov's theorem, the distribution of $W(g)$ and $\mathbf{E}^{-1}(g)$ with respect to $\mathrm{P}^{g}$ is the same as that of $W$ and $\mathrm{E}(-g)$ with respect to $\mathrm{P}$,

$$
\begin{aligned}
\mid \mathrm{E}( & \left.I_{\left\{\tau_{H}^{m}>T\right\}}-I_{\left\{\tau_{H}>T\right\}}\right)|=| \mathrm{E}\left(I_{\left\{S^{*} \geq H\right\}}\right)-\mathrm{E}\left(I_{\left\{S_{m}^{\prime} \geq H\right\}}\right) \mid \\
& =\left|\mathrm{E}^{\mu}\left(\mathrm{E}^{-1}(\mu) I_{\left\{(W(\mu))^{*} \geq L\right\}}\right)-\mathrm{E}^{\mu^{m}}\left(\mathrm{E}^{-1}\left(\mu^{m}\right) I_{\left\{\left(W\left(\mu^{m}\right)\right)_{m}^{*} \geq L\right\}}\right)\right| \\
& =\left|\mathrm{E}\left(\mathrm{E}(-\mu) I_{\left\{W^{*} \geq L\right\}}\right)-\mathrm{E}\left(\mathrm{E}\left(-\mu^{m}\right) I_{\left\{W_{m}^{*} \geq L\right\}}\right)\right| \\
& \leq \mathrm{E}\left(\left|\mathrm{E}(-\mu)-\mathrm{E}\left(-\mu^{m}\right)\right| I_{\left\{W^{*} \geq L\right\}}\right)+\mathrm{E}\left(\mathrm{E}\left(-\mu^{m}\right)\left|I_{\left\{W^{*} \geq L\right\}}-I_{\left\{W_{m}^{*} \geq L\right\}}\right|\right) \\
& =\varepsilon_{1}^{m}+\varepsilon_{2}^{m} .
\end{aligned}
$$

We estimate the first term. Note that $\mathrm{E}(-\mu)$ is the value of a solution of the stochastic differential equation $d X_{t}=\mu / \sigma d W_{t}$ at the point $T$, while $\mathrm{E}\left(-\mu^{m}\right)$ is the Euler approximation for this value. According to the standard estimate mentioned above,

$$
\varepsilon_{1}^{m} \leq \mathrm{E}\left(\left|\mathrm{E}(-\mu)-\mathrm{E}\left(-\mu^{m}\right)\right|\right) \leq C \sqrt{\Delta t} .
$$

To estimate $\varepsilon_{2}^{m}$ note that $I_{\left\{W^{*} \geq L\right\}}-I_{\left\{W_{m}^{*} \geq L\right\}}=I_{\left\{W^{*} \geq L, W_{m}^{*}<L\right\}}$; that is, one can assume that $W_{t_{i}}$ are bounded from above by the constant $L$ when estimating the mathematical expectation. We use the part summation in the formula for $\mathrm{E}\left(-\mu^{m}\right)$ :

$$
\int_{0}^{T} \frac{\mu_{t}^{m}}{\sigma} d W_{t}=\frac{1}{\sigma} \sum_{i=0}^{m-1} \mu_{t_{i}}\left(W_{t_{i+1}}-W_{t_{i}}\right)=\frac{1}{\sigma}\left(\mu_{t_{m-1}} W_{t_{m}}-\sum_{i=1}^{m-1} W_{t_{i}}\left(\mu_{t_{i}}-\mu_{t_{i-1}}\right)\right)
$$

and apply the Lipschitz condition for $\mu$. Then

$$
\varepsilon_{2}^{m} \leq C \mathrm{E}\left(\exp \left\{C \sup _{1 \leq i \leq m}\left(W_{t_{i}}\right)_{-}\right\} I_{\left\{W^{*} \geq L, W_{m}^{*}<L\right\}}\right),
$$

where $x_{-}=-\min \{x, 0\}$. Put $Z=\sup _{1 \leq i \leq m}\left(W_{t_{i}}\right)_{-}$. The random events

$$
A_{m}=\left\{W^{*} \geq L, W_{m}^{*}<L\right\} \quad \text { and } \quad B_{x}=\left\{\sup _{1 \leq i \leq m}\left(W_{t_{i}}\right)_{-}>x\right\}
$$

are negatively correlated. This becomes clear by looking at the conditional probabilities of these events given $W_{m}^{*}=y, y \in(x, L)$, whence the negative correlation is obvious for all $y$. The negativity of the correlation can also be shown by a straightforward calculation of the joint distribution density of $W_{t_{i}}, i=1, \ldots, m$, and $W^{*}$.

Now

$$
\begin{aligned}
\mathrm{E}\left(e^{C Z} I_{A}\right) & =\mathrm{E}\left(\left(1+\int_{0}^{\infty} C e^{C x} I_{\{Z>x\}} d x\right) I_{A_{m}}\right) \\
& =\mathrm{P}\left(A_{m}\right)+C \int_{0}^{\infty} e^{C x} \mathrm{P}\left(\{Z>x\} \cap A_{m}\right) d x \\
& \leq \mathrm{P}\left(A_{m}\right)+C \int_{0}^{\infty} e^{C x} \mathrm{P}\left(B_{x}\right) \mathrm{P}\left(A_{m}\right) d x=\mathrm{P}\left(A_{m}\right) \mathrm{E}\left(e^{C Z}\right) \leq C \mathrm{P}\left(A_{m}\right) .
\end{aligned}
$$


It remains to note that $\mathrm{P}\left(A_{m}\right) \sim C \sqrt{\Delta t}$ as $m \rightarrow \infty$ by [2, Theorem 2], whence $\varepsilon_{2}^{m} \leq C \sqrt{\Delta t}$.

Hence

$$
\left|V_{m}(H)-V(H)\right| \leq C \Delta t=O\left(\frac{1}{\sqrt{m}}\right)
$$

Remark 2.2. The estimate of the rate of convergence obtained in Theorem 2.1 is sharp, for example, for the Brownian motion with zero drift (see [2, Theorem 2]).

\section{Simulation}

Consider the following drift function:

$$
\mu_{t}= \begin{cases}\mu_{1}, & 0 \leq t<T / 2 \\ \mu_{2}, & T / 2 \leq t \leq T\end{cases}
$$

This function (and the corresponding interest rate $r_{t}$ ) does not satisfy the continuity condition (2.1) imposed in Theorem 2.1 on $\mu_{t}$. Analyzing the proof of Theorem 2.1, one can see that it holds if condition (2.1) is satisfied for $t=t_{i}, s \in\left[t_{i}, t_{i+1}\right)$. The latter property holds for the drift function $\mu_{t}$ defined above.

According to [4], if $X_{t}$ is a Brownian motion with the initial value $x$ and a constant drift coefficient $\mu$, then the joint distribution density of the distribution of the maximum $M_{t}$ in the interval $[0, t]$, the point $T_{t}$ of the maximum, and the value $X_{t}$ is given by

$$
\begin{aligned}
\mathrm{P}\left(X_{t}\right. & \left.\in d z, M_{t} \in d y, T_{t} \in d s\right) \\
& =\frac{(y-x)(y-z)}{\pi \sqrt{s^{3}(t-s)^{3}}} \exp \left(-\frac{(y-x)^{2}}{2 s}-\frac{(y-z)^{2}}{2(t-s)}-\mu(x-z)-\frac{\mu^{2} t}{2}\right) d z d y d s \\
& =: f_{t, x, \mu}(z, y, s) d z d y d s
\end{aligned}
$$

for $x \leq y$ and $z \leq y$, while it vanishes for $x>y$ or $z>y$. Let

$$
\nu(T)=\exp \left\{-\int_{0}^{T} r_{t} d t\right\}=\exp \left\{-\frac{T}{2}\left(\mu_{1}+\mu_{2}+\sigma^{2}\right)\right\} .
$$

Since $Z_{t}=\sigma^{-1} \ln S_{t}$ is a Brownian motion whose drift is such that $\nu_{1}=\mu_{1} / \sigma$ in $[0, T / 2)$ and $\nu_{2}=\mu_{2} / \sigma$ in $[T / 2, T]$, the fair price of a European call "up-and-out" option is given by

$$
\begin{aligned}
& V(H)=\mathrm{E}\left(\nu(T)\left(S_{T}-K\right)^{+} I_{\{\tau(H, S)>T\}}\right)=\nu(T) \mathrm{E}\left(\left(S_{T}-K\right)^{+} I_{\left\{\sup _{[0, T]} S_{t}<H\right\}}\right) \\
&= \nu(T) \mathrm{E}\left(\mathrm{E}\left(\left(S_{T}-K\right)^{+} I_{\left\{\sup _{[T / 2, T]} S_{t}<H\right\}} \mid \mathcal{F}_{T / 2}\right) I_{\left\{\sup _{[0, T / 2]} S_{t}<H\right\}}\right) \\
&= \nu(T) \mathrm{E}\left(\int_{0}^{T / 2} \int_{Z_{T / 2}}^{(\ln H) / \sigma} \int_{-\infty}^{y}\left(e^{\sigma z}-K\right)^{+}\right. \\
&\left.\times f_{T / 2, Z_{T / 2}, \nu_{2}}(z, y, s) d z d y d s I_{\left\{\sup _{[0, T / 2]} S_{t}<H\right\}}\right) \\
&=\nu(T) \int_{0}^{T / 2} \int_{Z_{0}}^{(\ln H) / \sigma} \int_{-\infty}^{v} f_{T / 2, Z_{0}, \nu_{1}}(x, v, u) \\
& \quad \times \int_{0}^{T / 2} \int_{x}^{(\ln H) / \sigma} \int_{-\infty}^{y}\left(e^{\sigma z}-K\right)^{+} f_{T / 2, x, \nu_{2}}(z, y, s) d z d y d s d x d v d u .
\end{aligned}
$$

The latter integral is hard to evaluate because of its high dimension. Nevertheless the integrals with respect to the variables $y$ and $v$ can be found analytically and expressed 
TABLE 1. The price $V(H)$ of a European call "up-and-out" option in the continuous model and the price $V_{m}(H)$ of the same option in the discrete model.

\begin{tabular}{|c|c|c|c|}
\hline$m$ & $V_{m}(H)$ & $V_{m}(H)-V(H)$ & $\left(V_{m}(H)-V(H)\right) \sqrt{m}$ \\
\hline 10 & 0.6808 & 0.2064 & 0.6527 \\
\hline 20 & 0.6183 & 0.1439 & 0.6435 \\
\hline 50 & 0.5686 & 0.0942 & 0.6661 \\
\hline 100 & 0.5476 & 0.0732 & 0.7321 \\
\hline 200 & 0.5198 & 0.0454 & 0.6421 \\
\hline 500 & 0.5073 & 0.0329 & 0.7356 \\
\hline 1000 & 0.4939 & 0.0195 & 0.6166 \\
\hline
\end{tabular}

in terms of the standard normal distribution function. However the expressions are quite complicated, so we do not provide them here. We only write the final bound for the integral.

Consider the following values of the parameters: $S_{0}=100, \sigma=0.1, K=100$, $H=105, T=0.2, \mu_{1}=0.1$, and $\mu_{2}=0.2$. Up to the accuracy $10^{-4}$, we have

$$
V(H)=0.4744 \text {. }
$$

To estimate the rate of convergence for the discrete time option prices we use the Monte Carlo method. We simulate 100,000 trajectories of the asset price $(50,000$ trajectories for $m=1,000)$ to estimate the mathematical expectation. The results are collected in Table 1. It is worthwhile mentioning that the option prices in discrete time models are greater than those in the continuous time model, and these prices decrease as the mesh of the partition increases. This is natural, since the set of moments when we check whether the price breaches the level is expanding as the number of points of the partition increases. Another observation is that the numerical experiment also confirms the rate of convergence obtained in Theorem 2.1 .

\section{Concluding Remarks}

We proved that the fair price of a barrier option in the discrete Black-Scholes model with varying drift coefficient converges to the corresponding price in the continuous time and that the rate of convergence is $O\left(m^{-1 / 2}\right)$, where $m$ is the number of transaction times in the discrete model.

\section{BIBLIOGRAPHY}

1. M. Broadie, P. Glasserman, and S. G. Kou, A continuity correction for discrete barrier options, Math. Finance 7 (1997), 325-349. MR1482707 (99k:90023)

2. M. Broadie, P. Glasserman, and S. G. Kou, Connecting discrete and continuous path-dependent options, Finance Stoch. 3 (1999), 55-82. MR.1805321 (2001k:91066)

3. P. Carmona, F. Petit, J. Pitman, and M. Yor, On the law of homogeneous functionals of the Brownian bridge, Studia Sci. Math. Hungar. 35 (1999), 445-455. MR 1762255 (2001f:60080)

4. E. Csáki, A. Földes, and P. Salminen, On the joint distribution of the maximum and its location for a linear diffusion, Ann. Inst. H. Poincaré Probab. Statist. 23 (1987), 179-194. MR 891709 (88k:60145)

5. P. Hörfelt, Extension of the corrected barrier approximation by Broadie, Glasserman, and Kou, Finance Stoch. 7 (2003), 231-243. MR 1968947 (2004b:91102)

6. P. E. Kloeden and E. Platen, Numerical Solution of Stochastic Differential Equations, Springer, Berlin, 1992. MR:1214374(94b:60069) 
7. S. G. Kou, On pricing of discrete barrier options, Statist. Sinica 13 (2003), 955-964. MR2026057 (2005a:62225)

8. R. C. Merton, Theory of rational option pricing, Bell J. Econom. and Management Sci. 4 (1973), 141-183. MR0496534 (58:15058)

Department of Probability Theory and Mathematical Statistics, Faculty for Mechanics and Mathematics, National Taras Shevchenko University, Academician Glushkov avenue, 6, KYIV 03127, UKRAINE

E-mail address: osoloveyko@univ.kiev.ua

Department of Probability Theory and Mathematical Statistics, Faculty for Mechanics and Mathematics, National Taras Shevchenko University, Academician Glushkov avenue, 6, KYIV 03127, UKRAINE

E-mail address: zhora@univ.kiev.ua

Received 7/MAR/2008

Translated by O. KLESOV 